\section{Hot white dwarfs}

SIR - THE discovery of a very hot hydrogen-rich white dwarf - RE1738+665 - in the Rosat survey (ref. 1) has generated much excitement (see the discussion in News and Views ${ }^{2}$ ). Although undoubtedly interesting, claims that it is a missing link are greatly exaggerated. Research over the past two years has shown that there are a substantial number of white dwarfs that help plug the so-called generation gap both those with and without ancient planetary nebulae. The lack of a clear division between the two groups is well illustrated by RE1738+665 itself: although it has been regarded as an isolated white dwarf, it does seem to have a planetary nebula associated with $\mathrm{it}^{3}$. There remain other problems with the current understanding of hot white dwarf evolution, but RE1738+665 is just one of several pieces of evidence showing that the elegant single-channel evolutionary model is long outdated.

A key feature of the single-channel model was that it could explain why $\mathrm{H}$-rich white dwarfs could be found only in two separate groups. These were the low- gravity central stars $\left(T_{\text {eff }}<80,000 \mathrm{~K}\right.$, surface gravity $\log g<5.5$; ref. 4$)$ and the highgravity white dwarfs which had cooled back below $70,000 \mathrm{~K}$ (ref. 5). The hottest known white dwarfs (with $70,000 \mathrm{~K}<T_{\text {eff }}$ $<170,000 \mathrm{~K}$ and surface gravity $\log g \sim 7$, for example PG1159-035; ref. 6) were all devoid of hydrogen. This has now changed. In their study of $\mathrm{H}$-rich white dwarfs found as central stars of planetary nebulae, Napiwotzki and Schönberner ${ }^{7,8}$ found 11 of 15 with $T_{\text {eff }}>70,000 \mathrm{~K}$, including NGC7293, the central star of the closest known planetary nebula, Sh2-216, and WDHS1, which may even be as hot as $200,000 \mathrm{~K}$. The parameters derived for three of these stars have been independently confirmed ${ }^{9,10}$. Higher temperatures are also being reported for known isolated white dwarfs, including PG1342+444 at $79,000 \mathrm{~K}, \mathrm{PG} 0939+262$ (Ton320) at 77,000 $\mathrm{K}$, and the remarkable $\mathrm{PG} 0948+534$, with

1. Barstow, M. A. et al. Mon. Not. R. astr. Soc. 271. 175-182 (1994).

3. Tweedy, R.W. \& Kwitter, K.B. Astrophys. J. 433, L93-L96 (1994).

4. Mendez, R. H. et al. Astr. Astrophys. 190, 113-136 (1988).

5. Holberg, J. B. et al. IAU Colloq. 114, 188-193 (1989). 437-461 (1991)

7. Napiwotzki, R. \& Schönberner, D. in White Dwarfs: Advances in Theory and Observation (ed. Barstow, M. A.) 99-105 (Kluwer, Dordrecht, 1993)

8. Napiwotzki, R thesis, Univ, Kiel (1993).

9. Bergeron, P. et al. Astrophys. J. 432, 305-325 (1994).

10. Liebert, J.W., Bergeron, P. \& Tweedy, R.W. Astrophys. J. 424, 817-822 (1994).

11. Barstow, M. A. et al. Mon. Not. R. astr. Soc. 264, 16-34 (1993).

12. Holberg, J. B. et al. Astrophys. J. 416, 806-819 (1993).

13. Fleming, T. A. Adv Space Res. 13, (12)7-13 (1993)

14. Wood, M. A. Astrophys. J. 386, 539-561 (1992).

15. Blöcker, T. \& Schönberner, D. Astr. Astrophys. 240.

5. Blöcker, T. \& Schô
L11-L13 (1990)
2. Shipman, H. L. Nature 372 318-319 (1994).

6. Werner, K., Heber, U. \& Hunger, K. Astr. Astrophys. 244,

16. Bergeron, P. et al. Astrophys. J. 394, 228-247 (1992)

$T_{\text {eff }}=130,000 \mathrm{~K}$ and $\log g=7.4$ (ref. 9).

The uniqueness of RE1738+665 lies in its being an EUV/soft X-ray source. It was a striking result of the all-sky survey performed by Rosat that essentially no H-rich white dwarfs above about $65,000 \mathrm{~K}$ were found ${ }^{11-13}$. Furthermore, those detected close to this upper limit have very steep short-wavelength cutoffs, together with ultraviolet spectra that are littered with many weak heavy-element lines ${ }^{12}$. Above this temperature, radiation pressure acting on the heavy elements appears to be sufficient to counteract the high gravities; their presence in the photosphere blocks out the short-wavelength emission. The sole exception to this is RE1738+665, which at $88,000 \mathrm{~K}$ is by far the hottest $\mathrm{H}$-rich white dwarf detected by Rosat. The reason is probably that it has a rather high mass. The value derived by Barstow et al. ${ }^{1}$ of $0.62 M_{\odot}$ is likely to be incorrect since they use evolutionary models that were designed for understanding old, cool white dwarfs ${ }^{14}$, which do not take into account the prior evolution from the AGB, and which is nec-

\section{Do long branches attract flies?}

SIR - We dispute Whiting and Wheeler's ${ }^{1}$ statement that $18 \mathrm{~S}$ ribosomal DNA sequence data support the Strepsiptera as sister-group to the Diptera (flies). Diptera and Strepsiptera group together in phylogenetic analyses, but we interpret this as an artefact resulting from the high substitution rates in their 18S rDNA sequences. Long-branch lineages - lineages with high substitution levels will cluster together with strong statistical support ${ }^{2,3}$.

We used complete $18 \mathrm{~S}$ rDNA insect sequences (Collembola, GenBank accession number Z26765; Hemiptera, U06478 and U06480; beetles, X07801 and X77786; Strepsiptera, X77784; Polistes wasp, X77785; Aedes mosquito, X57172; and Drosophila, M21017) and aligned them with partial sequences $(\sim 1,090$ nucleotides: several Hymenoptera, two Neuroptera, a flea and a scorpionfly $)^{4}$. Nucleotide substitution rates in 18S rDNA of flies and Strepsiptera were dramatically higher than in the other holometabolous insects (see figure), but these substitutions were not homologous with each other. Whether or not the strepsipteran and flies group with the scorpionfly and flea (which are close to the flies ${ }^{5}$ ) depends on the taxa and portion of the gene included in the analysis.

Phylogenetic placement of the Strepsiptera is an old problem which many entomologists are working to resolve ${ }^{5-7}$. Whiting and Wheeler ${ }^{1}$ may be right - essary for the hot $\operatorname{stars}^{15}$. Using the more accurate tracks in ref. 15 leads to a mass $\sim 0.7 M_{\odot}$ - high compared with the whitedwarf mass distribution, where $75 \%$ lie between 0.45 and $0.65 M_{\odot}$ (ref. 16). This higher gravity means that fewer heavy elements can be levitated to the surface layers, which increases the transparency to EUV/soft X-ray emission. High masses have also been reported for the pure- $\mathrm{H}$ which Barstow et al. ${ }^{1}$ refer to as possible progenitors of RE1738+665. Subdividing the H-rich objects on the basis of presence or absence of He is meaningful only insofar as it highlights the importance of the mass of the remnant. RE1738+665 is a fascinating object, but its importance to understanding white-dwarf evolution should not be overstated.

Richard W. Tweedy

Steward Observatory,

University of Arizona,

Tucson,

Arizona 85721 ,

USA central stars, EGB1 and WDHS1 (ref. 8), flies and strepsipterans might be close relatives. In our analyses the $18 \mathrm{~s}$ rDNA data provide no evidence to support this conclusion. However, because Whiting and Wheeler have not made their data available, it has not been possible to examine
Bootstrap consensus phylogram from parsimony (PAUP 3.1, 2,000 replicates, 889 steps) showing the long branches of the Diptera and Strepsiptera.

the opposing evidence. Instead, the grouping of the Strepsiptera and Diptera in the 18S rDNA tree appears to be an excellent example of long-branch attraction resulting from the violation of the molecular clock for this gene.

David Carmean, Bernard J. Crespi

Department of Biological Sciences,

Simon Fraser University, Burnaby,

British Columbia V5A 1S6, Canada

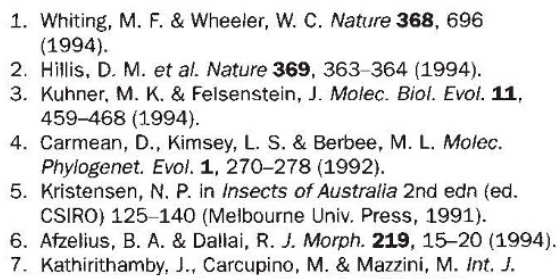

